\title{
Beyond Biomimetics: Towards Insect/Machine Hybrid Controllers for Space Applications
}

\author{
Antonella Benvenuto ${ }^{\text {a,* }}$, Fabrizio Sergi ${ }^{\text {a }}$, Giovanni Di Pino ${ }^{\text {a }}$, Tobias Seidl ${ }^{\text {b }}$, \\ Domenico Campolo $^{a}$, Dino Accoto ${ }^{a}$ and Eugenio Guglielmelli ${ }^{a}$ \\ ${ }^{\text {a }}$ CIR — Laboratory of Biomedical Robotics and Biomicrosystems, Università Campus Bio-Medico, \\ Via Álvaro del Portillo, 21-00128 Rome, Italy \\ ${ }^{\mathrm{b}}$ Advanced Concepts Team, European Space Agency, Keplerlaan 1, 2201 AZ Noordwijk, \\ The Netherlands
}

Received 4 August 2008; accepted 28 October 2008

\begin{abstract}
Robots for space applications require a level of autonomy while operating in highly unstructured environments that current control architectures cannot manage successfully. We investigated including predeveloped insect brain tissue into the control architecture of space exploratory vehicles. A doubly hybrid controller is proposed that hinges around the 'insect-in-a-cockpit' concept towards an evolution of the classical deliberative/reactive paradigm, featuring a biological (insect brain) high-level deliberative module coupled with low-level reactive behaviors embedded in a robot. The proposed concept, its design methodology and functional description of the submodules are presented, along with a preliminary feasibility assessment mainly derived from an in-depth review of the state of the art.

(c) Koninklijke Brill NV, Leiden and The Robotics Society of Japan, 2009
\end{abstract}

Keywords

Biomimetics, hybrid robot control architectures, insect navigation, neural interfaces, biomechatronics

\section{Introduction}

Facing the challenges of autonomous space exploration, the range of future automated mission vehicles strongly correlates with the capability of the controller to successfully integrate a whole range of decision capabilities. Nowadays, working devices reproduce a range of isolated components of complex behaviors, such as flight stabilization, obstacle avoidance, altitude control, directional control and landmark recognition [1-3]. However, some tasks, such as navigation without drift

\footnotetext{
* To whom correspondence should be addressed. E-mail: a.benvenuto@unicampus.it
} 
towards a distant goal, reaction to unexpected perturbations, memorization of new experiences and learning of new strategies, require a level of complexity that current control architectures are still far from being able to successfully manage.

Fundamental analogies exist between the behaviors that insects exhibit and the basic skills that one would expect from autonomous robots in space. Insects such as bees, ants and cockroaches have become particularly appealing models for investigation in the context of biomimetic robotics since they have optimized navigational mechanisms in terms of simplicity and robustness $[4,5]$. In this context, the use of insect intelligence could create an intermediate type of mission bridging between purely robotic and human controlled missions.

This paper presents the conceptualization and the design methodology of a doubly hybrid controller that includes a living insect in a cockpit. The insect intelligence controls the robotic platform when decision making and/or cognition (i.e., reasoning) are required. The interfacing between the insect and the robotic platform can be accomplished by means of natural and neural interfaces. Well beyond biomimetics, insect/machine hybrid systems represent a new challenging approach merging biomimetics, neurophysiology, ethology and microengineering.

Given the inherent technological challenges, some simplifying assumptions are required in this study so as to address the most challenging goals from the strict robotics research viewpoint:

(i) We focus on the use of pre-developed living tissue and do not consider in vitro development of biological neuronal networks, since we want to profit from the highly elaborate behaviors observed in living animals.

(ii) We assume that it is feasible to keep alive and functional the animal brain tissue (or the whole insect) for a period of time appropriate for space missions.

(iii) The robotic platform can be designed according to good practice taught by biomimetics. In particular, we will not consider any specific control issues that can be already solved with a smart (e.g., biomimetic) state-of-the-art design.

In order to reach our design goal, an interdisciplinary approach has been followed which moved from an in-depth review at the intersection of insect-inspired robotics, insect behaviors and insect/robot interfacing to end up with a detailed proposal of the novel hybrid control architecture, as reported in the following sections of this paper.

\section{Biomimetics Versus Biology: Towards Hybridity}

\subsection{Insect-Inspired Robotics}

The biologically inspired approach to the design of robotic agents intends to refer to natural evolution of species as an endless source of design solutions, which are optimized for a given ecological niche. Although the focus of traditional biologically inspired robotics has been mainly on neural modeling for mimicking animal 
behaviors, recent developments in the field have centred on the concepts of embodiment, which is the reciprocal and dynamic coupling between the brain, body and environment [6]. This approach has led to the development of new robotic agents that are more suitable for dealing with the real world and perform successfully in uncertain conditions. In recent years, many robotics laboratories have worked on the design of bio-inspired agents trying to put in practice these design principles, so that a wide collection of animal-like robots can be reported at the present time. We will mainly focus in the following on robotic solutions concerning bio-inspired locomotion.

In recent years, research effort has been devoted to the development of cockroach-inspired walking and running hexapod robots $[7,8]$ with robust selfstabilization intrinsic capabilities that allow locomotion even on irregular terrain. This is achieved by exploiting the dynamic coupling between the legs and the ground, which enormously simplifies the control.

The capabilities of adaptation to different terrains have also been demonstrated in snake-like robots, by varying either ground friction [9] or slope [10].

Furthermore, flying microrobots inspired by flies have been designed [11, 12]. These robots can generate the necessary high-dimensional wing trajectories by a smart, bio-inspired design of wings kinematics and passive dynamic properties, thus simplifying the number of actuators required to accomplish the task of flapping flight. The beneficial generation of multiple degrees of freedom (d.o.f.) trajectories with a single d.o.f. of actuation has been also achieved in a fish robot called Wanda [13]. In this realization the unbalanced buoyancy of the structure is exploited to map, through the dynamic interaction with the environment, the wiggling frequency, amplitude and waveform of the tail with the speed, attitude and heading direction of the robot, respectively.

The case of underwater walking has been investigated for developing a robot inspired on the American lobster [14]; such a system reproduces many salient properties of lobster locomotion, ranging from the ability to adapt to a complex variety of bottom types to the ability to perform 'tactile' navigation through flow and contact sensors.

A very interesting feature of the locomotion of some animal forms is the gait transition capability, which allows energy optimization and adaptation to different types of environments. Gait transition is also very interesting from the perspective of highlighting the underlying neural mechanisms at its base. As an example, the transition from swimming to walking of salamanders is of great relevance for studying vertebrate evolution through the analysis of spinal locomotor circuits [15]. A model simulating a central pattern generator has been implemented in a salamander-like amphibious robot [16] as a system of coupled non-linear oscillators, which receives a simple high-level command signal as input, triggering and modulating the transition among swimming, serpentine crawling and walking. 
Another very interesting feature well addressed by bio-inspired robotics is climbing on vertical surfaces $[17,18]$, which has been obtained in robotic agents by using a micropatterned fibrillar dry adhesive inspired by the gecko's foot morphology.

These examples show that a bio-inspired design approach can improve the locomotive performances of mobile robots in terms of maneuverability and adaptation to uncertain situations. Higher-level tasks like navigation in unknown environments [1], social behaviors and division of labour [19, 20], which require some abstraction and decision making, have also been addressed in autonomous mobile robots with a bio-inspired approach.

The results obtained by research in this field are promising from the perspective of improving the capabilities of autonomous robots in uncertain conditions. Despite that, reproducing a global animal behavior in a mobile robot through a bio-inspired design is still far from being successful. For this purpose, we consider the option of interfacing the robot with a pre-developed intelligence worth being pursued, from the perspective of exploiting the basic insect cognition abilities to directly implement deliberative functionality in a robotic artefact.

\subsection{Main Features of Insect Behaviors}

The addition of a pre-developed intelligence can improve robot performances for complex high-level task management. Among several animal species, fundamental analogies exist between the behaviors that insects exhibit and the basic skills that we would expect from autonomous robots in space.

In recent years, several studies have demonstrated that the insect neuronal system is capable of dealing flexibly and adaptively with a wide range of ecologically relevant problems; the possibility of a central integration that horizontally combines different domain-specific modules to form new behaviors and new solutions has also been considered [21, 22]. Two areas of the insect brain are commonly associated with multi-sensory convergence: the mushroom bodies (MB), which play a major role during spatio-temporal sensory processing and learning [23], and the central complex (CX), which is involved in (pre-)motor processing, higher locomotion control, including initiation and modulation of behavior, goal-directed motion and path integration [24]. In most insect species, e.g., bees and ants, the compass direction can be gained from celestial cues (allothetic cues), while different approaches are used for distance estimation, e.g., bees record optic flow, while ants and cockroaches mainly use proprioceptive information (idiothetic cues) [25]. In consequence, data from different sensors need to be integrated sense-fully in the brain in order to acquire the desired vector information. For the 'insect-in-a-cockpit' scenario, which will be presented in detail in Section 3, we considered honeybees to be the most suitable species, since their odometer works via visual cues and it is well characterized [26]. Moreover, honeybees have unique cognitive capabilities and their nervous system is easily accessible [21, 22]. 


\subsection{Current Approaches Towards Hybridity}

Current approaches towards hybridity are presented with a twofold aim. On one side, we aim to better underline the novelty aspects of the different proposed approaches; on the other, we review the state of the art in order to establish what is already feasible and what will likely be feasible in the near future.

There are several different technological possibilities to interface living tissue with robots. The first choice is between non-invasive cockpit-like interfaces, where the intact animal's sensors are confronted with natural stimuli, and invasive interfaces. The invasive technique allows choosing between neural interfaces, in which information is transmitted through neurons, or non-neural approaches (e.g., through muscle stimulating/recording electromyography electrodes). Non-neural interfacing is a more practical approach compared to neural interfacing, but it presents some drawbacks. The amount of information that can be transferred via a non-neural interface is lower and this may hinder the detection of complex behaviors. Moreover, the electric current intensity required to directly stimulate a muscle is about 10-fold higher than that needed to stimulate the nerve to obtain the same movement $(1 \mathrm{~mA}$ to $100 \mu \mathrm{A})$ [27].

Neural interfaces roughly divide into three groups: electrodes stimulate and monitors (i) cultured neurons, (ii) a pre-grown brain tissue removed from the rest of the body or (iii) a brain tissue still connected to the body. Neuronal cultures have been grown in two- and three-dimensional architectures, and have demonstrated astonishing performance $[28,29]$; however, it remains questionable if they will become capable of resolving high-level control issues, since their random arrangement does not correspond to any anatomical substrate for any complex function requiring interaction with the external environment.

The possibility to use brain-computer interfaces (BCIs) with insects is a very promising field to investigate. One of the main challenges of invasive brainmachine interfacing lies in the surgical procedure and all issues related to it, such as mechanical and electrical stability of the interface along the time of implantation.

Currently, one of the most favored techniques for neural interfaces in insect BCIs uses flexible polymer-based multi-electrodes arrays. They can be bent around a nerve or around an insect appendage, enabling multi-unit recording and stimulation without inhibiting the animal's locomotion [30]. In the last decade, integrated circuitry and micro-fabrication technologies have led to a drastic reduction of size, allowing the development of systems including a neural probe, battery and microcontroller weighing less than $500 \mathrm{mg}$, which can be implanted in cockroaches [31].

As it relates to natural interfaces, which are widely used in insect neuroethology research, programmable visual arenas allow reproducing optic flow to the insect [32], while force sensors [33] and magnetic coils [34] facilitate recording of motor responses to presented stimuli.

At the current state of the art, there are only a few reports on bidirectional insectrobot interfacing: the cockroach robot [35] and the moth robot [36]. The cockroach robot, implemented at Irvine University, has a bidirectional natural interface con- 
sisting of eight distance proximity sensors, LED panels and a modified trackball. The setup executes motor commands as decoded from movements of an insect positioned on the trackball; environmental sensory data are acquired through proximity sensors and encoded into a light stimulus presented to the cockroach. The system is limited to fleeting behavior (one of the few behaviors bypassing the CX), which is simply triggered by the very predictable stimulus-response reflexes. Therefore, the hybrid system does not exploit the high-level autonomous behaviors, such as navigation and exploration, afforded by the insect's brain.

The moth robot displays a combination of both natural and neural interfacing. It was developed at the University of Arizona. In this system, both natural and neural interfaces are implemented; the former through a continuous optic flow provided by a 14-inch $(35.6 \mathrm{~cm})$ high revolving wall painted with vertical stripes, the latter by recording the electrical activity of visual motion neurons. In order to implement this approach, the moth is immobilized inside a plastic tube mounted on a wheeled robot. The robot itself can turn left or right, according to neural signals translated by a computer. The system can be useful to characterize visual motion detection neurons, but it is not reported if a closed-loop sensing and action behavior is achieved.

The reported examples demonstrate the technological feasibility of insect/ machine neural and natural interfacing. Considering the present level of knowledge about neural interfaces in insects, it does not appear possible to transfer all superior control functions from the machine to the insect, especially when connected through a neural interface exclusively. Thus, we followed the approach of combining neural and natural interfaces in order to achieve multi-modal communication, and hence a redundancy of information exchange.

\section{Doubly Hybrid Controller}

\subsection{Proposed Hybrid Control Architecture}

As far as hybrid insect/machine control architectures are concerned, we propose to set the degree of hybridity as a trade-off between low-level and high-level behaviors. Given the whole set of desirable behaviors of an autonomous robotic platform, we classified as 'high-level' those behaviors requiring decision-making and/or cognitive capabilities (e.g., path planning), whilst all the remaining behaviors (e.g., obstacle avoidance) will be considered as 'low-level'. In practice, low-level behaviors will be those that can be carried out by state-of-the-art robotic platforms without an active involvement of the insect. Since extraterrestrial missions do not usually require fast reactions, several approaches are pursuable in order to have the robot perform the necessary low-level behaviors.

For space applications, we are mainly interested in navigation and exploration tasks with compensating reactions towards unexpected perturbations. An imaginary scenario would include a hybrid controlled exploratory rover navigating through various landscapes and returning to a fixed relay station on a regular basis. The operating environment, hence, is in principle very different from the natural en- 


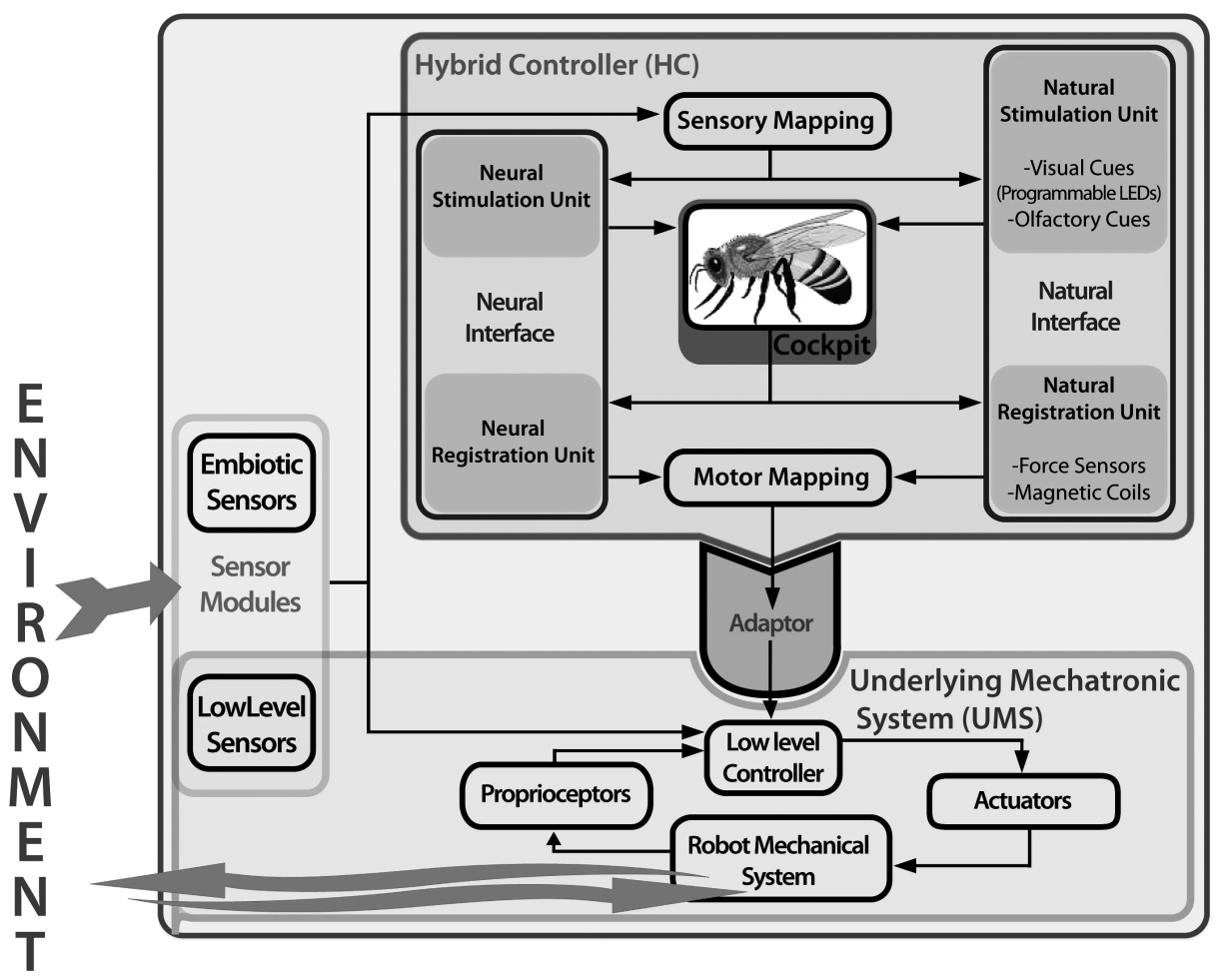

Figure 1. Scheme of a robotic platform including the hybrid control architecture.

vironment where an insect may live. The inputs from the environment have to be translated into nature-analog signals before being provided to the insect. This is definitely a critical step towards the exploitation of pre-developed biological intelligence. The next section will describe how this can be achieved.

According to these assumptions and starting from fundamental control architecture paradigms $[35,36]$, a novel doubly hybrid control architecture, including both biological/artificial modules and deliberative/reactive behaviors, has been hypothesized [39]. The architecture proposed here comprises four functional modules: (i) the sensor modules, (ii) the hybrid controller (HC), (iii) the adaptor and (iv) the underlying mechatronic system (UMS). Figure 1 shows the schematic of a robotic platform including the proposed doubly hybrid controller and its submodules.

The sensor modules consist of both the low-level sensors and embiotic (from Greek prefix 'em-' meaning 'in, into' and 'biotic' meaning 'pertaining to life') sensors. The low-level sensors are included in the UMS (e.g., proximity sensors, inertial modules, wheel/leg encoders and wheel/leg slide sensors for self-stabilization). The embiotic sensors complement the set of UMS sensors and may include vision systems, temperature sensors, light polarization sensors, etc., according to the selected insect and the navigation/exploration tasks. The neologism 'embiotic' highlights that these sensors mainly pertain to the insect perceptual capabilities and 


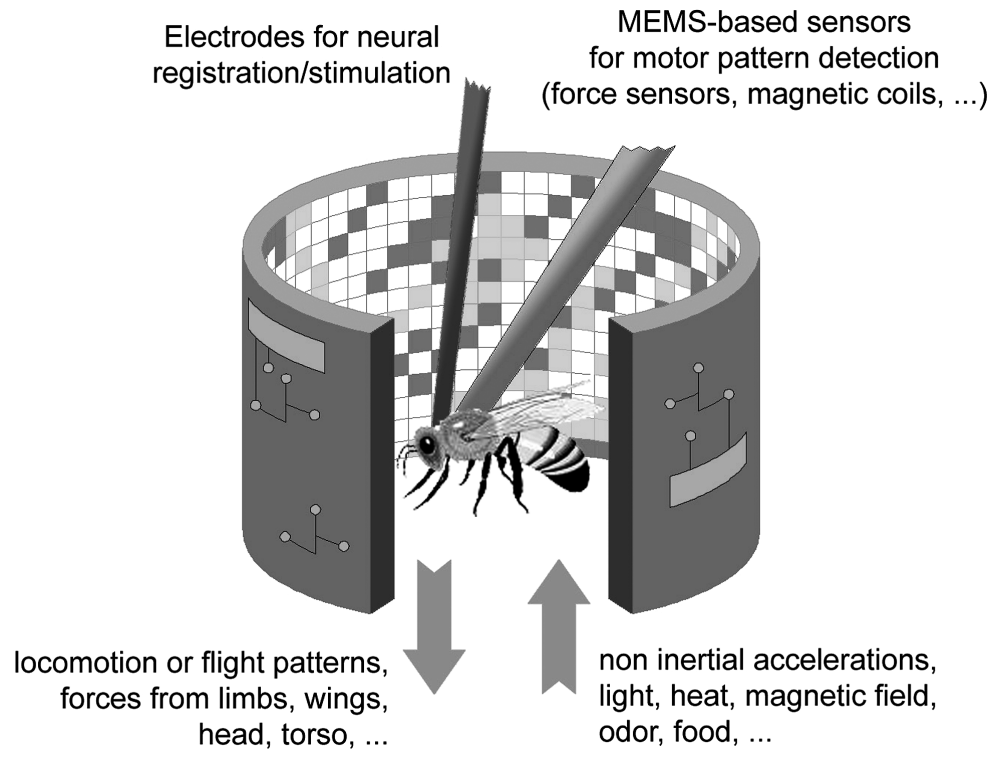

Figure 2. Insect-in a-cockpit concept.

are used to provide the necessary inputs to the insect for the implementation of those high-level tasks, that can be accomplished when the insect intelligence acts through the hybrid controller.

The hybrid controller is composed of the mapping modules (sensory and motor), the interfacing modules (neural and natural) and the cockpit. The sensory and motor mapping modules are the core elements of the proposed architecture and are detailed in Section 3.2. In particular, our main interest is to exploit insect predeveloped skills to tackle high-level tasks by a proper mapping that gives inputs as natural-like as possible.

The mapped stimuli from a new environment (e.g., the Martian soil) are received from the insect tethered in the cockpit both via natural and neural interfaces. For example, the tethered insect may receive input stimuli via LED panels and via implanted electrodes, while the triggered motor responses can be detected by using different types of sensors, such as force sensors and EMG electrodes. A simplified representation of the 'insect-in-a-cockpit' concept is shown in Fig. 2.

The insect's motor commands are processed by an adaptor module, which gives input to the low-level controller of the UMS. The adaptor enhances the HC interoperability with different hardware platforms (UMSs): since it is the only module that directly transmits data from the $\mathrm{HC}$ to the UMS, the $\mathrm{HC}$ can be used with different UMSs, tailored to the specific application scenario, by only changing the adaptor module.

Finally, the UMS includes:

- Proprioception sensors used for information related to the robot internal state (in particular, sensors for energy and failure monitoring). 
- Low-level sensors used to implement low-level behaviors (e.g., locomotion).

- A low-level controller that properly weights inputs from sensor modules, proprioceptors and the adaptor, thus allowing the correct driving of the robotic platform.

From a design methodological perspective, some basic steps have been systematized for guiding the development of a doubly hybrid controller for a robotic system. First, the specific task of interest has to be selected. The second step consists in choosing the most appropriate insect species that can be identified by means of a table that scores:

- The ability of the insect to respond to allothetic cues.

- The feasibility of triggering an insect response by means of natural interfaces.

- Insect's cognitive capabilities.

- Technological feasibility of neural interfaces.

Once the choice of the insect is made, it is necessary to define the level of hybridity (third step), i.e., for a given task it should be decided which high-level behaviors should be implemented by the insect and which ones (low-level behaviors) should be allocated to the UMS. In the case that the hybrid controller has to be implemented starting from a specific existing robot, the degree of hybridity can be derived only following an attentive analysis of its actual capabilities.

The fourth step consists in choosing which signals should be conveyed to the neural stimulation path and which to the natural stimulation path.

As a fifth step, the selection of the UMS has to be accomplished (only if an existing specific system was not already selected $a$ priori). This choice basically depends on the available state-of-the-art robotic technologies. As an example, when considering navigation and exploration tasks, locomotion (either wheeled or legged) on rough terrains is reliably achieved by state-of-the-art robots. Proper vehicles have been specifically designed to cope with the nature of the Martian soil, e.g., asperities, presence of sand/dust, local gravity and radiation $[2,40]$. These are 'details' that the insect should not get involved with. Note that more than one choice might actually be available to the designer.

Finally, the sixth step consists in the submodule design.

\subsection{Sensory and Motor Mapping}

The dynamics of complex behaviors such as navigation or exploration can be considered a product of more basic behavioral elements, i.e., attraction and repellence, in relation to specific features of the environment. Attractors could be rendered with food sources, the nest or a prey, while repellers could be represented by predators and obstacles.

Navigation and exploration skills rely on the ability of insects to create internal maps of the environment. As part of the environment, both attractors and repellers 
Table 1.

Elementary behaviors and their triggers in exploration/navigation tasks differentiated by the predictability of the trigger's position

\begin{tabular}{lll}
\hline & $\begin{array}{l}\text { Attractor behavior } \\
\text { (trigger) }\end{array}$ & $\begin{array}{l}\text { Repeller behavior } \\
\text { (trigger) }\end{array}$ \\
\hline Static & pursuit (nest) & $\begin{array}{l}\text { avoidance (obstacle) } \\
\text { fleeting (predator) }\end{array}$ \\
\hline
\end{tabular}

need to be taken into account in the mapping process and, therefore, can be classified as static, if their position is fixed with respect to the map, or dynamic, if their position within the map varies with time. For example, the nest can be considered as a static attractor, while a prey would be a dynamic one. Similarly, an obstacle such as a big rock would be a static repeller, while a predator would represent a dynamic one. These behaviors are schematized in Table 1.

Each input resulting from embiotic sensors is then presented to the animal following the scheme of Table 1. Although each single behavior per se might be considered as a reflex, simply triggered by the presence of the repeller or of the attractor, the simultaneous presentation of competing sensory cues would elicit a 'decision-making' process of the insect. From this perspective, multi-modal sensory inputs are more likely to elicit the higher level and autonomous behaviors.

In addition to the external stimuli representing the environment to the animal, internal stimuli, e.g., metabolic needs, may also have an influence on decisions to be taken. As an example, these needs can be used to trigger through olfactory cues the insect behavior of navigation towards a nest, which can be mapped into the robot task of routing towards a recharge site. In such a scenario, schematized in Table 2, olfactory cues can be combined with visual cues (optic flow and polarization cues) to provide the insect with the information concerning heading and distance towards the virtual nest. Simultaneously, repellers occurring in the external environment can be presented visually to the insect, allowing the elicitation of avoidance or fleeting behaviors. The insect's torso, head and wing movements are measured and mapped into motor commands to robot actuators, which generate locomotion in the direction suggested by the insect. When the robot reaches the recharge site, the insect can be rewarded with real food.

Moreover, typical features of insect navigation such as the use of landmarks or visual route learning can be exploited to extend autonomy by, for example, removing typical drift errors occurring in robotic platforms without external reference systems.

The flow of information to and from the insect occurs via a combination of both natural and neural interfaces in order to achieve both redundancy and robustness. The communication established has to assure enough stability and bandwidth to allow correctly driving the robot. 
Table 2.

Schematization of navigation towards the nest

\begin{tabular}{|c|c|c|c|}
\hline $\begin{array}{l}\text { Information from } \\
\text { the environment }\end{array}$ & Sensory mapping & Insect behavior & Motor mapping \\
\hline $\begin{array}{l}\text { Location of the } \\
\text { recharge site }\end{array}$ & $\begin{array}{l}\text { primitive needs (food in the } \\
\text { nest) are presented to the } \\
\text { insect by means of olfactory } \\
\text { cues in the cockpit }\end{array}$ & $\begin{array}{l}\text { navigation towards } \\
\text { the 'nest' is } \\
\text { triggered }\end{array}$ & $\begin{array}{l}\text { scheduling activities } \\
\text { related to energy level } \\
\text { of the robotic platform }\end{array}$ \\
\hline $\begin{array}{l}\text { Presence of } \\
\text { attractors/repellers }\end{array}$ & $\begin{array}{l}\text { the environment is reproduced } \\
\text { to the insect by means of } \\
\text { optic flow and polarization } \\
\text { cues }\end{array}$ & $\begin{array}{l}\text { torso/head/wing } \\
\text { movements are } \\
\text { generated towards } \\
\text { the virtual nest, } \\
\text { avoiding repellers } \\
\text { in the real world }\end{array}$ & $\begin{array}{l}\text { robot actuators are } \\
\text { driven following insect } \\
\text { movements; the robot } \\
\text { explores the } \\
\text { environment and } \\
\text { creates a map }\end{array}$ \\
\hline $\begin{array}{l}\text { Arrival at the } \\
\text { recharge site }\end{array}$ & $\begin{array}{l}\text { the insect is rewarded with } \\
\text { water/food }\end{array}$ & $\begin{array}{l}\text { the insect feeds } \\
\text { itself }\end{array}$ & the robot is recharged \\
\hline
\end{tabular}

\section{Conclusions}

The success of automated mission vehicles strongly correlates with the capability of the control architecture to successfully integrate a whole range of decision parameters. The addition of pre-developed insect intelligence in robotic platforms could create an intermediate type of mission bridging between purely robotic and human controlled missions. In this context we investigated how to integrate 'insect intelligence' into the control architecture of a hypothetical exploratory vehicle including the modalities to exploit the full potential of insect intelligence.

The architecture presented here delegates higher level behaviors, such as decision making and planning, to the insect, while low-level tasks are executed by the robotic platform. Its double hybridity, i.e., biological/artificial as well as deliberative/reactive, allows for managing concurrent behaviors such as accurate goal navigation and drifting compensation in parallel. The metaphor of the proposed interface is a cockpit, where the tethered insect receives both natural and neural stimuli.

Sensory-motor mapping is conceptualized in order to match the pre-developed navigation and exploration skills of the insect with the operating environment. An adaptor module is proposed to process the insect motor response and to give proper inputs to the low-level controller of the robotic platform, thus enhancing the hybrid controller properties in terms of interoperability with different hardware platforms (UMSs).

Even though much work is required for the development and validation of the proposed control architecture (e.g., design and fabrication of the 'cockpit' and of the neural bidirectional interface; definition of a suitable performance/benchmarking metrics in order to assess the achieved performance and compare it to that of state- 
of-the-art autonomous agents), the discussed concepts represent a first step for the integration of 'pre-developed intelligence' in space robots.

\section{Acknowledgements}

This work was supported by the ESA Ariadna Medium Study 'Machine/Animal Hybrid Controllers for Space Applications (07/6301)'.

\section{References}

1. M. O. Franz and H. A. Mallot, Biomimetic robot navigation, Robotics Autonomous Syst. 30, 133153 (2000).

2. S. Thakoor, N. Cabrol, N. Lay, J. Chahl, D. Soccol, B. Hine and S. Zornetzer, Review: the benefits and applications of bioinspired flight capabilities, J. Robotics Syst. 20, 687-706 (2003).

3. N. Franceschini, Visual guidance based on optic flow: a biorobotic approach, J. Phys. 98, 281-292 (2004).

4. R. Wehner, Desert ant navigation: how miniature brains solve complex tasks, J. Comp. Physiol. A 189, 579-588 (2003).

5. M. Dacke and M. V. Srinivasan, Honeybee navigation: distance estimation in the third dimension, J. Exp. Biol. 210, 845-853 (2007).

6. R. Pfeifer, M. Lungarella and F. Iida, Self-organization, embodiment, and biologically inspired robotics, Science 318, 1088-1093 (2007).

7. J. G. Cham, J. K. Karpick and M. R. Cutkosky, Stride period adaptation for a biomimetic running hexapod, Int. J. Robotic Res. 23, 1-13 (2004).

8. R. Altendorfer, N. Moore, H. Komsuoglu, M. Buehler, H. B. Brown, D. McMordie, U. Saranli, R. Full and D. Koditschek, RHex: a biologically inspired hexapod runner, Autonomous Robots 11, 207-213 (2001).

9. K. Inoue, T. Sumi and S. Ma, CPG-based control of a simulated snake-like robot adaptable to changing ground friction, in: Proc. IEEE/RSJ Int. Conf. on Intelligent Robots and Systems (IROS), San Diego, CA, pp. 1957-1962 (2007).

10. S. Ma, N. Tadokoro and K. Inoue, Influence of gradient of a slope to optimal locomotion curves of a snake-like robot, Adv. Robotics 20, 413-428 (2006).

11. R. J. Wood, The first takeoff of biologically inspired at-scale robotic insect, IEEE Trans. Robotics 24, 341-346 (2008).

12. R. S. Fearing, S. Avadhanula, D. Campolo, M. Sitti, J. Yan and R. Wood, A micromechanical flying insect thorax, in: Neurotechnology for Biomimetic Robots, D. R. Ayers (Ed.), pp. 469-480. MIT Press, Cambridge, MA (2000).

13. R. Pfeifer, F. Iida and G. Gomez, Morphological computation for adaptive behavior and cognition, Int. Congr. Ser. 1291, 22-29 (2006).

14. J. Ayers and J. Witting, Biomimetic approaches to the control of underwater walking machines, Philos. Trans. R. Soc. A 365, 273-295 (2007).

15. A. J. Ijspeert, A. Crespi and J. M. Cabelguen, Simulation and robotic studies of salamander locomotion, Neuroinformatics 3, 171-195 (2005).

16. A. Ijspeert, A. Crespi, D. Ryczko and J. M. Cabelguen, From swimming to walking with a salamander robot driven by a spinal cord model, Science 315, 1416-1420 (2007).

17. M. Setti, Microscale and nanoscale robotics systems: caracteristics, state of the art, and grand challenges, IEEE Robotics Automat. Mag. 14, 53-60 (2007). 
18. S. Kim, M. Spenko, S. Trujillo, B. Heyneman, D. Santos and M. R. Cutkosky, Smooth vertical surface climbing with directional adhesion, IEEE Trans. Robotics 24, 65-74 (2008).

19. T. Fong, I. Nourbakhsh and K. Dautenhahn, A survey of socially interactive robots, Robotics Autonomous Syst. 42, 143-166 (2003).

20. M. Waibel, D. Floreano, S. Magnenat and L. Keller, Division of labour and colony efficiency in social insects: effects of interactions between genetic architecture, colony kin structure and rate of perturbations, Proc. Roy. Soc. B 273, 1815-1823 (2006).

21. R. Menzel and M. Giurfa, Cognitive architecture of a mini-brain: the honeybee, Trends Cognit. Sci. 5, 62-71 (2001).

22. R. Menzel, G. Leboulle and D. Eisenhardt, Small brains, bright minds, Cell 124, 237-239 (2006).

23. R. Menzel, Searching for the memory trace in a mini-brain, the honeybee, Learn. Mem. 8, 53-62 (2001).

24. H. Vitzthum, M. Muller and U. Homberg, Neurons of the central complex of the locust Schistocerca gregaria are sensitive to polarized light, J. Neurosci. 22, 1114-1125 (2002).

25. T. Seidl, M. Knaden and R. Wehner, Desert ants: is active locomotion a prerequisite for path integration?, J. Comp. Physiol. A 192, 1125-1131 (2006).

26. M. V. Srinivasan and R. L. Gregory, How bees exploit optic flow: behavioural experiments and neural models, Philos. Trans. Biol. Sci. 337, 253-259 (1992).

27. J. Mavoori, B. Millard, J. Longnion, T. Daniel and C. Diorio, A miniature implantable computer for functional electrical stimulation and recording of neuromuscular activity, in: Proc. IEEE Int. Workshop on Biomedical Circuits and Systems, Singapore, pp. S1/7-S1/13 (2004).

28. A. Novellino, P. D’Angelo, L. Cozzi, M. Chiappalone, V. Sanguineti and S. Martinoia, Connecting neurons to a mobile robot: an in vitro bidirectional neural interface, Comp. Intell. Neurosci. 2007, $1-13$ (2007).

29. S. M. Potter, D. A. Wagenaar, R. Madhavan and T. B. DeMarse, Long-term bidirectional neuron interfaces for robotic control, and in vitro learning studies, in: Proc. 25th Int. Conf. IEEE EMBS, Cancun, pp. 3690-3693 (2003).

30. A. J. Spence, K. B. Neeves, D. Murphy, S. Sponberg, B. R. Land, R. R. Hy and M. S. Isaacson, Flexible multielectrodes can resolve multiple muscles in an insect appendage, J. Neurosci. Methods 159, 116-124 (2007).

31. H. Sato, C. Berry, B. Casey, G. Lavella, Y. Yao, J. VanderBrooks and M. Maharbiz, A cyborg beetle: insect flight control through an implantable, tetherless microsystem, in: Proc. of MEMS, Tucson, AZ, pp. 164-167 (2008).

32. M. B. Reiser and M. H. Dickinson, A modular display system for insect behavioral neuroscience, J. Neurosci. Methods 167, 127-139 (2008).

33. Y. Sun, S. N. Fry, D. P. Potasek, D. J. Bell and B. J. Nelson, Characterizing fruit fly flight behavior using a microforce sensor with a new comb-drive configuration, J. Microelectron. Syst. 14, 4-11 (2005).

34. C. Schilstra and J. H. Van Haeren, Blowfly flight and optic flow, thorax kinematics and flight dynamics, J. Exp. Biol. 202, 1481-1490 (1999).

35. http://www.conceptlab.com/roachbot

36. http://neuromorph.ece.arizona.edu

37. R. A. Brooks, A robust layered control system for a mobile robot, IEEE J. Robotics Automat. 2, 14-23 (2006).

38. R. Murphy, Introduction to AI Robotics. MIT Press, Cambridge, MA (2000). 
39. A. Benvenuto, F. Sergi, G. Di Pino, D. Campolo, D. Accoto, E. Guglielmelli and T. Seidl, Conceptualization of an insect/machine hybrid controllers for space applications, in: Proc. IEEE Int. Conf. on Biomedical Robots and Biomechanics, Scottsdale, AZ, pp. 306-310 (2008).

40. R. Beard, D. J. Lee, M. Quigley, S. Thakoor and S. Zornetzer, A new approach to future Mars missions using bioinspired techonology innovation, J. Aer. Comp. Inf. Commun. 2, 65-91 (2005).

\section{About the Authors}

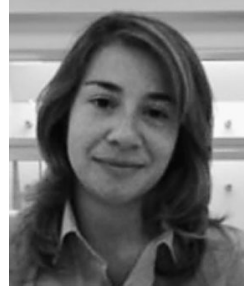

Antonella Benvenuto is currently a Post-Doc Fellow in Bioengineering at the Università Campus Bio-Medico in Rome, Italy. She received her Laurea Degree in Electric Engineering from the University of Pisa, in 1998, and the PhD in Microsystems Engineering from the University of Rome 'Tor Vergata', in 2002. From 2002 to 2007, she had a postdoctoral position at Bio-MEMS research group of FBK-irst Research Institute in Trento, Italy. In January 2008, she joined the Biomedical Robotics and Biomicrosystems Lab of Campus Bio-Medico University, Rome, Italy. Her research activity is focused on the development of microsystems for biomedical applications, e.g., chemical-based diagnostic microsystems and neural invasive interfaces. She has co-authored more than 20 papers, appearing in international journals and conference proceedings.

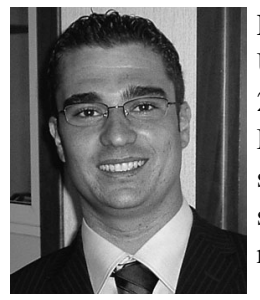

Fabrizio Sergi received his BS and MS degree in Biomedical Engineering from Università Campus Bio-Medico, Rome, in 2005 and 2007, respectively. In January 2008, he started his PhD studies in Biomedical Robotics at Università Campus BioMedico, Laboratory of Biomedical Robotics and Biomicrosystems, under the supervision of Professor Eugenio Guglielmelli. His is primarily interested in novel interaction schemes between biological systems and robots, with particular regard to biomedical robots for rehabilitation or assitive purposes.

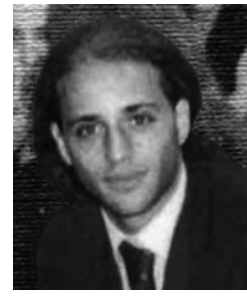

Giovanni Di Pino received the Medical Degree (cum laude and honor mention) in 2003 at Campus Bio-Medico University, Rome. He worked in Italian and American Laboratories mainly involved in studying the mechanisms and molecules of neurodevelopment, apoptosis and neurophysiological intracellular recording. In 2001 and 2002, he was an Internal Research Fellow in the Neurobiology Department at the University of Pittsburgh. His current fields of interest include neuroscience and bio-engineering, and all the possible matches. In 2006, he started a $\mathrm{PhD}$ program in Bioengineering primarily involved in developing bidirectional brain-computer interfaces to control sensorized cybernetic prostheses and to improve bio-electronic hybridity.

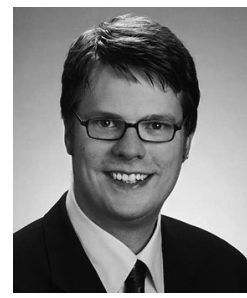

Tobias Seidl studied technical biology and biomimetics in Saarbruecken (Germany) including a half-year stay at BMW group in Munich. At that time he focused on quantitative atomic force microscopy characterizing the material properties of the arthropod cuticle. For his $\mathrm{PhD}$ he went to Zurich (Switzerland) and Mahares (Tunisia) to study the path integration mechanisms of desert ants together with Professor Ruediger Wehner. Currently he is a Research Fellow at the Advanced Concepts Team of the European Space Agency in Noordwijk (The Netherlands), focussing on the transfer of insect neuronal systems to space applications. Within the Ariadna framework, he aims to sensitize the biomimetic community for space applications. He works and publishes both with the biological and the engineering community. 


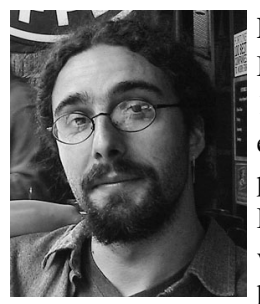

Domenico Campolo is currently a Senior Researcher at Università Campus BioMedico in Rome, Italy. He received his Laurea Degree from the University of Pisa, in 1998, and the Diploma Degree from Scuola Superiore Sant'Anna, in 1999. In 2002, he earned his PhD (Diploma di Perfezionamento) in Micro-Engineering from Scuola Superiore Sant'Anna, Pisa, while working at MiTech Lab (currently, the CRIM Lab). During the Fall 1998 he was working at the EECS Department of ZheJiang University, HangZhou, PRC, as a Visiting Graduate Student. In the period 2000-2003, he was at UC-Berkeley, USA, as a Visiting Scholar and, after 2002, as a Post-Doc working on the MFI (Micromechanical Flying Insect) project. Since October 2003, he has been working at Campus Bio-Medico University where he is involved in both teaching and research. His research interests include mechatronic technologies with application to the new emerging fields of phenomics and neuro-developmental engineering, in both animal and human models; biomimetic microrobotics, including: design, fabrication, development and control of biologically inspired smart actuators and sensors; micro/nano-manipulation; and micro/nano-fabrication.

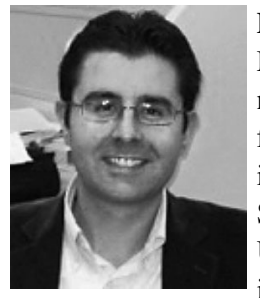

Dino Accoto is Assistant Professor of Biomedical Engineering at Campus BioMedico University, Rome, Italy. He received the Laurea Degree in Mechanical Engineering from the University of Pisa (cum laude), in 1998, the diploma in Engineering from the Scuola Sant'Anna (cum laude), in 1999, and the PhD degree in Biomedical Robotics from the Scuola Sant'Anna in 2002 (cum laude). From October 2001 to September 2002, he was a Visiting Scholar at the RPL-Lab, Stanford University (CA, USA). From 2003 to 2007, he was an Assistant Professor in Biomedical Engineering at the Scuola Sant'Anna. Since 2004, he has been with the Biomedical Robotics and Biomicrosystems Lab at Campus Bio-Medico University, Rome. His research interest is the development of electromechanical systems for biorobotics and biomedicine using multi-scale and multi-physics approaches. He has co-authored more than 40 papers, appearing in international journals and conference proceedings, and is co-author of five patents.

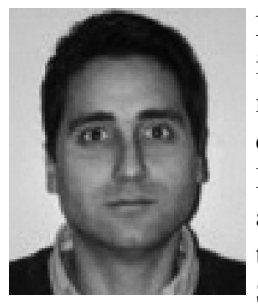

Eugenio Guglielmelli received the Laurea Degree (Master) in Electronics Engineering, and the $\mathrm{PhD}$ in Elctronics, Telecommunications and Computer Science (Biomedical Robotics track) from the University of Pisa, Italy, in 1991 and 1995. He is currently Associate Professor of Bioengineering at Università Campus Bio-Medico, Rome, Italy, where he serves as the Head of the Laboratory of Biomedical Robotics and Biomicrosystems. From 1991 to 2004, he worked with Professor Paolo Dario at the Advanced Robotics Technology \& Systems Laboratory (ARTS Lab) of the Scuola Superiore Sant'Anna, Pisa, Italy, that he directed from 2002 to 2004 . His main current research interests are in the fields of human-centred robotics, biomechatronic design and biomorphic control of robotic systems, and in their application to robot-mediated motor therapy, assistive robotics and neurorobotics. He is author/co-author of more than 150 papers appearing in peer-reviewed international journals, conference proceedings and books. He currently serves as Associate Editor of the IEEE Robotics and Automation Magazine and on the Editorial Board of the international journal on Applied Bionics and Biomechanics. He has been Guest Co-Editor of the Special Issues on Rehabilitation Robotics of the IEEE Transactions on Robotics and of the International Journal Autonomous Robots, and also of the Special Issue on Robotics Platforms for Neuroscience of the RSJ international journal Advanced Robotics. He is principal investigator/partner of several national and international projects in the area of biomedical robotics. He is member of the IEEE Robotics \& Automation Society, IEEE Engineering in Medicine \& Biology Society and Society for Neuroscience. He is currently Associate Vice-President for Technical Activities of the IEEE Robotics \& Automation Society (RAS) and he also served as Co-chair of the Technical Committee on Rehabilitation and Assistive Robotics (2004-2007) and as RAS Secretary (2002-2003) of the same society. He was/is member of the Organizing Committees of HURO1998, ICAR2003, IROS2004, IFAC/SYROCO2006, ICRA2007 and ROMAN2009, and of the Programme Committees of several international conferences, workshops and symposia. 